\title{
Aryeh Kofsky, Serge Ruzer, in collaboration with Reuven Kiperwasser. Reshaping Identities in Late Antique Syria-Mesopotamia. Christian and Jewish Hermeneutics and Narrative Strategies
}

Florence Jullien

\section{OpenEdition}

\section{Journals}

Édition électronique

URL : http://journals.openedition.org/abstractairanica/44623

DOI : $10.4000 /$ abstractairanica. 44623

ISBN : 1961-960X

ISSN : 1961-960X

Éditeur :

CNRS (UMR 7528 Mondes iraniens et indiens), Éditions de l'IFRI

\section{Référence électronique}

Florence Jullien, « Aryeh Kofsky, Serge Ruzer, in collaboration with Reuven Kiperwasser. Reshaping Identities in Late Antique Syria-Mesopotamia. Christian and Jewish Hermeneutics and Narrative Strategies », Abstracta Iranica [En ligne], Volume 37-38-39 | 2018, document 25, mis en ligne le 30 décembre 2018, consulté le 29 septembre 2020. URL : http://journals.openedition.org/abstractairanica/44623 DOI : https://doi.org/10.4000/abstractairanica.44623

Ce document a été généré automatiquement le 29 septembre 2020.

Tous droits réservés 


\title{
Aryeh Kofsky, Serge Ruzer, in collaboration with Reuven
} Kiperwasser. Reshaping Identities in Late Antique Syria-Mesopotamia. Christian and Jewish Hermeneutics and Narrative Strategies

\author{
Florence Jullien
}

\section{RÉFÉRENCE}

Aryeh Kofsky, Serge Ruzer, in collaboration with Reuven Kiperwasser. Reshaping Identities in Late Antique Syria-Mesopotamia. Christian and Jewish Hermeneutics and Narrative Strategies. Piscataway: Gorgias Press, 2016, 276 p. (Judaism in Context 19), ISBN 978-1-4632-0590-4.

1 Les études présentées dans ce volume portent sur la question des rencontres interculturelles et des échanges entre communautés juives et chrétiennes (dans la diversité de leurs expressions théologiques) d'une part, et entre celles-ci et d'autres traditions religieuses (paganisme et zoroastrisme essentiellement) dans l'Antiquité tardive. Cette problématique est surtout abordée sous l'angle de la formation et de la défense des identités religieuses en Syrie, mais aussi en Mésopotamie dans le contexte perse sassanide. Les questions de définition de frontières ou de délimitation des champs de collaboration, leurs variations ou leur suppression selon les contextes, soustendent les réflexions. Une première partie a trait à ce que les AA. appellent un « clivage » au sein du christianisme syriaque entre les composantes grecque et syriaque de la réception de la doctrine de Théodore de Mopsueste («Theodore of Mopsuestia 
and the Greek-Syriac Divide of Syrian Christianity "). Cette disjonction est étudiée à travers des thèmes aussi variés que l'anthropologie et la sotériologie (chapitre 3), le développement de la personne (chapitre 2), les diverses formes de christologie (chapitre 1: "Transformed Christology in Response to Crises »). Une deuxième partie aborde le cadre culturel des relations intercommunautaires, spécialement entre juifs et chrétiens ("Syriac Christians and Jews in a Syro-Mesopotamian Cultural Setting »). On notera que les principaux éléments du chapitre $5:$ «Syriac and rabbinic narratives on Zoroastrian oral culture : the case of a Persian convert » ont déjà été développés par Reuven Kiperwasser dans une étude de 2014 ( To convert a Persian and to teach him the holy scriptures. A Zoroastrian proselyte in Rabbinic and Syriac Christian narratives ", dans G. Herman (ed.), Jews, Christians and Zoroastrians. Religious dynamics in a Sasanian context, Piscataway, 2014, p. 91-127). Les chapitres 6: «Broader cultural patterns in Jewish and Christian discourse » et $7:$ «Competition for sacred space : Bar Sauma's Vita and rabbinic traditions" montrent, à travers quelques cas d'espèce, l'intérêt d'une étude comparative entre les sources chrétiennes syriaques et les sources rabbiniques babyloniennes. Sans évoquer des dépendances ou des influences directes, les points de convergence ainsi dégagés soulignent, pour les AA., l'existence de modèles littéraires caractéristiques des discours patristiques et rabbiniques. Voir dans ce même ordre leur étude de 2015 : S. Ruzer, R. Kiperwasser, «Syriac Christians and Babylonian Jewry: Narratives and Identity Shaping in a Multi-Religious Setting ", dans B. BittonAshkelony, T. de Bruyn, C. Harrison (ed.), Patristic Studies in the Twenty-First Century. Proceedings of an International Conference to Mark the 50th Anniversary of the International Association of Patristic Studies, Turnhout, 2015, p. 421-440). L'ouvrage a l'intérêt de croiser des exemples issus de sources de provenances communautaires très diverses, et de les remettre en contexte, suggérant des interactions interculturelles et des communications entre ces différents groupes religieux. L'ouvrage s'achève par une bibliographie détaillée et des index utiles.

\section{AUTEURS}

\section{FLORENCE JULLIEN}

CNRS, Mondes iranien et indien, Paris 